\title{
Pacific
}

Journal of

Mathematics

\section{MULTIGRADED FUJITA APPROXIMATION}

SHIN-YAO JOW

Volume $251 \quad$ No. 2

June 2011 


\title{
MULTIGRADED FUJITA APPROXIMATION
}

\author{
SHIN-YAO JOW
}

The original Fujita approximation theorem states that the volume of a big divisor $D$ on a projective variety $X$ can always be approximated arbitrarily closely by the self-intersection number of an ample divisor on a birational modification of $X$. One can also formulate it in terms of graded linear series as follows: Let $W_{\bullet}=\left\{W_{k}\right\}$ be the complete graded linear series associated to a big divisor $D$, where

$$
W_{k}=H^{0}\left(X, O_{X}(k D)\right) .
$$

For each fixed positive integer $p$, define $W_{\bullet}^{(p)}$ to be the graded linear subseries of $W_{\bullet}$ generated by $W_{p}$ :

$$
W_{m}^{(p)}= \begin{cases}0 & \text { if } p \nmid m, \\ \operatorname{Image}\left(S^{k} W_{p} \rightarrow W_{k p}\right) & \text { if } m=k p .\end{cases}
$$

Then the volume of $W_{\bullet}^{(p)}$ approaches the volume of $W_{\bullet}$ as $p \rightarrow \infty$. We will show that, under this formulation, the Fujita approximation theorem can be generalized to the case of multigraded linear series.

\section{Introduction}

Let $X$ be an irreducible variety of dimension $d$ over an algebraically closed field $\boldsymbol{K}$, and let $D$ be a (Cartier) divisor on $X$. When $X$ is projective, the following limit, which measures how fast the dimension of the section space $H^{0}\left(X, \mathscr{O}_{X}(m D)\right)$ grows, is called the volume of $D$ :

$$
\operatorname{vol}(D)=\operatorname{vol}_{X}(D)=\lim _{m \rightarrow \infty} \frac{h^{0}\left(X, O_{X}(m D)\right)}{m^{d} / d !} .
$$

One says that $D$ is big if $\operatorname{vol}(D)>0$. It turns out that the volume is an interesting numerical invariant of a big divisor [Lazarsfeld 2004a, Section 2.2.C], and it plays a key role in several recent works in birational geometry [Tsuji 2000; Boucksom et al. 2004; Hacon and McKernan 2006; Takayama 2006].

Keywords: Fujita approximation, multigraded linear series, Okounkov body. 
When $D$ is ample, one can show that $\operatorname{vol}(D)=D^{d}$, the self-intersection number of $D$. This is no longer true for a general big divisor $D$, since $D^{d}$ may even be negative. However, Fujita [1994] showed that the volume of a big divisor can always be approximated arbitrarily closely by the self-intersection number of an ample divisor on a birational modification of $X$. This theorem, known as Fujita approximation, has several implications for the properties of volumes, and is also a crucial ingredient in [Boucksom et al. 2004] (see [Lazarsfeld 2004b, Section 11.4] for more details).

Lazarsfeld and Mustaţă [2009] (henceforth [LM]) recently obtained, among other things, a generalization of Fujita approximation to graded linear series. Recall that a graded linear series $W_{\bullet}=\left\{W_{k}\right\}$ on a (not necessarily projective) variety $X$ associated to a divisor $D$ consists of finite dimensional vector subspaces

$$
W_{k} \subseteq H^{0}\left(X, \mathscr{O}_{X}(k D)\right)
$$

for each $k \geq 0$, with $W_{0}=\boldsymbol{K}$, such that

$$
W_{k} \cdot W_{\ell} \subseteq W_{k+\ell}
$$

for all $k, \ell \geq 0$. Here the product on the left denotes the image of $W_{k} \otimes W_{\ell}$ under the multiplication map $H^{0}\left(X, \mathscr{O}_{X}(k D)\right) \otimes H^{0}\left(X, \mathscr{O}_{X}(\ell D)\right) \rightarrow H^{0}\left(X, O_{X}((k+\ell) D)\right)$. In order to state the Fujita approximation for $W_{\bullet}$, they defined, for each fixed positive integer $p$, a graded linear series $W_{\bullet}^{(p)}$ which is the subgraded linear series of $W_{\bullet}$ generated by $W_{p}$ :

$$
W_{m}^{(p)}= \begin{cases}0 & \text { if } p \nmid m, \\ \operatorname{Im}\left(S^{k} W_{p} \rightarrow W_{k p}\right) & \text { if } m=k p .\end{cases}
$$

Then under mild hypotheses, they showed that the volume of $W_{\bullet}^{(p)}$ approaches the volume of $W_{\bullet}$ as $p \rightarrow \infty$. See [LM, Theorem 3.5] for the precise statement, as well as [LM, Remark 3.4] for how this is equivalent to the original statement of Fujita when $X$ is projective and $W_{\bullet}$ is the complete graded linear series associated to a big divisor $D$ (that is, $W_{k}=H^{0}\left(X, O_{X}(k D)\right)$ for all $\left.k \geq 0\right)$.

The goal of this note is to generalize the Fujita approximation theorem to multigraded linear series. We will adopt the following notation from [LM, Section 4.3]: Let $D_{1}, \ldots, D_{r}$ be divisors on $X$. For $\vec{m}=\left(m_{1}, \ldots, m_{r}\right) \in \mathbb{N}^{r}$, write $\vec{m} D=$ $\sum m_{i} D_{i}$, and put $|\vec{m}|=\sum\left|m_{i}\right|$.

Definition. A multigraded linear series $W_{\vec{\bullet}}$ on $X$ associated to the $D_{i}$ consists of finite-dimensional vector subspaces

$$
W_{\vec{k}} \subseteq H^{0}\left(X, \mathrm{O}_{X}(\vec{k} D)\right)
$$


for each $\vec{k} \in \mathbb{N}^{r}$, with $W_{\overrightarrow{0}}=\boldsymbol{K}$, such that

$$
W_{\vec{k}} \cdot W_{\vec{m}} \subseteq W_{\vec{k}+\vec{m}},
$$

where the multiplication on the left denotes the image of $W_{\vec{k}} \otimes W_{\vec{m}}$ under the natural map

$$
H^{0}\left(X, \bigcirc_{X}(\vec{k} D)\right) \otimes H^{0}\left(X, \bigcirc_{X}(\vec{m} D)\right) \rightarrow H^{0}\left(X, \bigcirc_{X}((\vec{k}+\vec{m}) D)\right) .
$$

Given $\vec{a} \in \mathbb{N}^{r}$, denote by $W_{\vec{a}}, \bullet$ the singly graded linear series associated to the divisor $\vec{a} D$ given by the subspaces $W_{k \vec{a}} \subseteq H^{0}\left(X, \bigcirc_{X}(k \vec{a} D)\right)$. Then put

$$
\operatorname{vol}_{W_{\bullet}}(\vec{a})=\operatorname{vol}\left(W_{\vec{a}, \bullet}\right)
$$

(assuming that this quantity is finite). It will also be convenient for us to consider $W_{\vec{a}, \bullet}$ when $\vec{a} \in \mathbb{Q}_{\geq 0}^{r}$, given by

$$
W_{\vec{a}, k}= \begin{cases}W_{k \vec{a}} & \text { if } k \vec{a} \in \mathbb{N}^{r}, \\ 0 & \text { otherwise. }\end{cases}
$$

Our multigraded Fujita approximation, similar to the singly graded version, is going to state that (under suitable conditions) the volume of $W_{\overrightarrow{0}}$ can be approximated by the volume of the following finitely generated submultigraded linear series of $W_{\overrightarrow{0}}$ :

Definition. Given a multigraded linear series $W_{\overrightarrow{0}}$ and a positive integer $p$, define $W_{\vec{\bullet}}^{(p)}$ to be the submultigraded linear series of $W_{\vec{\bullet}}$ generated by all $W_{\vec{m}_{i}}$ with $\left|\vec{m}_{i}\right|=p$, or concretely,

$$
W_{\vec{m}}^{(p)}=\left\{\begin{array}{cl}
0 & \text { if } p \nmid|\vec{m}|, \\
\sum_{\substack{\left|\vec{m}_{i}\right|=p \\
\vec{m}_{1}+\cdots+\vec{m}_{k}=\vec{m}}} W_{\vec{m}_{1}} \cdots W_{\vec{m}_{k}} & \text { if }|\vec{m}|=k p .
\end{array}\right.
$$

We now state our multigraded Fujita approximation when $W_{\vec{\bullet}}$ is a complete multigraded linear series, since this is the case of most interest and allows for a more streamlined statement. The Remark on page 335 points out what assumptions on $W_{\vec{\bullet}}$ are actually needed in the proof.

Theorem. Let $X$ be an irreducible projective variety of dimension d, and let $D_{1}$, $D_{2}, \ldots, D_{r}$ be big divisors on $X$. Let $W_{\vec{\bullet}}$ be the complete multigraded linear series associated to the $D_{i}$, namely

$$
W_{\vec{m}}=H^{0}\left(X, O_{X}(\vec{m} D)\right)
$$


for each $\vec{m} \in \mathbb{N}^{r}$. Then given any $\varepsilon>0$, there exists an integer $p_{0}=p_{0}(\varepsilon)$ having the property that if $p \geq p_{0}$, then

$$
\left|1-\frac{\operatorname{vol}_{W_{\vec{\bullet}}(p)}(\vec{a})}{\operatorname{vol}_{W_{\vec{\bullet}}}(\vec{a})}\right|<\varepsilon
$$

for all $\vec{a} \in \mathbb{N}^{r}$.

\section{Proof of the Theorem}

The main tool in our proof is the theory of Okounkov bodies developed systematically in [Lazarsfeld and Mustață 2009]. Given a graded linear series $W_{\bullet}$ on a $d$-dimensional variety $X$, its Okounkov body $\Delta\left(W_{\bullet}\right)$ is a convex body in $\mathbb{R}^{d}$ that encodes many asymptotic invariants of $W_{\bullet}$, the most prominent one being the volume of $W_{\bullet}$, which is precisely $d$ ! times the Euclidean volume of $\Delta\left(W_{\bullet}\right)$. The idea first appeared in Okounkov's papers [1996; 2003] in the case of complete linear series of ample line bundles on a projective variety. Later it was further developed and applied to much more general graded linear series by Lazarsfeld and Mustaţă [2009] and also independently by Kaveh and Khovanskii [2008; 2009].

Proof of the Theorem. Let $T=\left\{\left(a_{1}, \ldots, a_{r}\right) \in \mathbb{R}_{\geq 0}^{r} \mid a_{1}+\cdots+a_{r}=1\right\}$, and let $T_{\mathbb{Q}}$ be the set of all points in $T$ with rational coordinates. The fraction inside (1) is invariant under scaling of $\vec{a}$ due to homogeneity, hence it is enough to prove (1) for $\vec{a} \in T_{\mathbb{Q}}$.

Let $\Delta\left(W_{\overrightarrow{0}}\right) \subseteq \mathbb{R}^{d} \times \mathbb{R}^{r}$ be the global Okounkov cone of $W_{\overrightarrow{0}}$ as in [LM, Theorem 4.19], and let $\pi: \Delta\left(W_{\vec{\bullet}}\right) \rightarrow \mathbb{R}^{r}$ be the projection map. For each $\vec{a} \in T$, write $\Delta\left(W_{\vec{\bullet}}\right)_{\vec{a}}$ for the fiber $\pi^{-1}(\vec{a})$. Define in a similar fashion the convex cone $\Delta\left(W_{\vec{\bullet}}^{(p)}\right)$ and the convex bodies $\Delta\left(W_{\vec{\bullet}}^{(p)}\right)_{\vec{a}}$. By [LM, Theorem 4.19],

$$
\Delta\left(W_{\vec{\bullet}}\right)_{\vec{a}}=\Delta\left(W_{\vec{a}, \bullet}\right) \quad \text { for all } \vec{a} \in T_{\mathbb{Q}} .
$$

Although [LM, Theorem 4.19] requires $\vec{a}$ to be in the relative interior of $T$, here we know that (2) holds even for those $\vec{a}$ in the boundary of $T$ because the big cone of $X$ is open and $W_{\overrightarrow{0}}$ was assumed to be the complete multigraded linear series. By the singly graded Fujita approximation, $\operatorname{vol}\left(W_{\vec{a}}, \bullet\right)$ can be approximated arbitrarily closely by $\operatorname{vol}\left(W_{\vec{a}, \bullet}^{(p)}\right)$ if $p$ is sufficiently large. (Here by $W_{\vec{a}, \bullet}^{(p)}$ we mean $W_{\bullet}^{(p)}$ restricted to the $\vec{a}$ direction, which certainly contains $\left(W_{\vec{a}, \bullet}\right)^{(p)}$.) Hence given any finite subset $S \subset T_{\mathbb{Q}}$ and any $\varepsilon^{\prime}>0$, we have

$$
\operatorname{vol}\left(\Delta\left(W_{\vec{\bullet}}^{(p)}\right)_{\vec{a}}\right) \geq \operatorname{vol}\left(\Delta\left(W_{\vec{\bullet}}\right)_{\vec{a}}\right)-\varepsilon^{\prime} \quad \text { for all } \vec{a} \in S
$$

as soon as $p$ is sufficiently large.

Because the function $\vec{a} \mapsto \operatorname{vol}\left(\Delta\left(W_{\overrightarrow{0}}\right)_{\vec{a}}\right)$ is uniformly continuous on $T$, given any $\varepsilon^{\prime}>0$, we can partition $T$ into a union of polytopes with disjoint interiors 
$T=\bigcup T_{i}$, in such a way that the vertices of each $T_{i}$ all have rational coordinates, and on each $T_{i}$ we have a constant $M_{i}$ such that

$$
M_{i} \leq \operatorname{vol}\left(\Delta\left(W_{\vec{\bullet}}\right)_{\vec{a}}\right) \leq M_{i}+\varepsilon^{\prime} \quad \text { for all } \vec{a} \in T_{i} .
$$

Let $S$ be the set of vertices of all the $T_{i}$. Then as we saw in the end of the previous paragraph, as soon as $p$ is sufficiently large we have

$$
\operatorname{vol}\left(\Delta\left(W_{\vec{\bullet}}^{(p)}\right)_{\vec{a}}\right) \geq \operatorname{vol}\left(\Delta\left(W_{\vec{\bullet}}\right)_{\vec{a}}\right)-\varepsilon^{\prime} \quad \text { for all } \vec{a} \in S .
$$

We claim that this implies

$$
\operatorname{vol}\left(\Delta\left(W_{\vec{\bullet}}^{(p)}\right)_{\vec{a}}\right) \geq \operatorname{vol}\left(\Delta\left(W_{\vec{\bullet}}\right)_{\vec{a}}\right)-2 \varepsilon^{\prime} \quad \text { for all } \vec{a} \in T_{\mathbb{Q}} .
$$

To show this, it suffices to verify it on each of the $T_{i}$. Let $\vec{v}_{1}, \ldots, \vec{v}_{k}$ be the vertices of $T_{i}$. Then each $\vec{a} \in T_{i}$ can be written as a convex combination of the vertices: $\vec{a}=\sum t_{j} \vec{v}_{j}$ where each $t_{j} \geq 0$ and $\sum t_{j}=1$. Since $\Delta\left(W_{\vec{\bullet}}^{(p)}\right)$ is convex, we have

$$
\Delta\left(W_{\vec{\bullet}}^{(p)}\right)_{\vec{a}} \supseteq \sum t_{j} \Delta\left(W_{\vec{\bullet}}^{(p)}\right)_{\vec{v}_{j}},
$$

where the sum on the right means the Minkowski sum. By (3) and (4), the volume of each $\Delta\left(W_{\bullet}^{(p)}\right)_{\vec{v}_{j}}$ is at least $M_{i}-\varepsilon^{\prime}$, hence by the Brunn-Minkowski inequality [Kaveh and Khovanskii 2008, Theorem 5.4], we have

$$
\operatorname{vol}\left(\Delta\left(W_{\vec{\bullet}}^{(p)}\right)_{\vec{a}}\right) \geq M_{i}-\varepsilon^{\prime} \quad \text { for all } \vec{a} \in T_{i} \cap T_{\mathbb{Q}} .
$$

This combined with (3) shows that (5) is true on $T_{i} \cap T_{\mathbb{Q}}$, hence it is true on $T_{\mathbb{Q}}$ since the $T_{i}$ cover $T$.

Since (1) follows from (5) by choosing a suitable $\varepsilon^{\prime}$, the proof is complete.

Remark. In the statement of the Theorem we assume that $W_{\overrightarrow{0}}$ is the complete multigraded linear series associated to big divisors. But in fact since the main tool we used in the proof is the theory of Okounkov bodies established in [Lazarsfeld and Mustață 2009], in particular [LM, Theorem 4.19], the really indispensable assumptions on $W_{\overrightarrow{0}}$ are the same as those in [LM] (which they called Conditions $\left(\mathrm{A}^{\prime}\right)$ and $\left(\mathrm{B}^{\prime}\right)$, or $\left(\mathrm{C}^{\prime}\right)$ ). The only place in the proof where we invoke that we are working with a complete multigraded linear series is the sentence right after (2), where we want to say that (2) holds not only in the relative interior of $T$ but also in its boundary. Hence if $W_{\overrightarrow{0}}$ is only assumed to satisfy Conditions $\left(\mathrm{A}^{\prime}\right)$ and $\left(\mathrm{B}^{\prime}\right)$, or $\left(\mathrm{C}^{\prime}\right)$, then given any $\varepsilon>0$ and any compact set $C$ contained in $T \cap \operatorname{int}\left(\operatorname{supp}\left(W_{\vec{\bullet}}\right)\right)$, there exists an integer $p_{0}=p_{0}(C, \varepsilon)$ such that if $p \geq p_{0}$ then

$$
\operatorname{vol}_{W_{\vec{\bullet}}(p)}(\vec{a})>\operatorname{vol}_{W_{\vec{\bullet}}}(\vec{a})-\varepsilon
$$

for all $\vec{a} \in C \cap T_{\mathbb{Q}}$. 


\section{Acknowledgments}

The author would like to thank Robert Lazarsfeld for raising this question during an email correspondence.

\section{References}

[Boucksom et al. 2004] S. Boucksom, J.-P. Demailly, M. Paun, and T. Peternell, "The pseudoeffective cone of a compact Kähler manifold and varieties of negative Kodaira dimension”, preprint, 2004. arXiv math/0405285

[Fujita 1994] T. Fujita, "Approximating Zariski decomposition of big line bundles", Kodai Math. J. 17:1 (1994), 1-3. MR 95c:14053 Zbl 0814.14006

[Hacon and McKernan 2006] C. D. Hacon and J. McKernan, "Boundedness of pluricanonical maps of varieties of general type”, Invent. Math. 166:1 (2006), 1-25. MR 2007e:14022 Zbl 1121.14011

[Kaveh and Khovanskii 2008] K. Kaveh and A. Khovanskii, "Convex bodies and algebraic equations on affine varieties", preprint, 2008. arXiv 0804.4095

[Kaveh and Khovanskii 2009] K. Kaveh and A. Khovanskii, "Newton convex bodies, semigroups of integral points, graded algebras and intersection theory", preprint, 2009. arXiv 0904.3350

[Lazarsfeld 2004a] R. Lazarsfeld, Positivity in algebraic geometry, I: Classical setting: line bundles and linear series, Ergebnisse der Math. und ihrer Grenzgebiete (3) 48, Springer, Berlin, 2004. MR 2005k:14001a Zbl 1093.14501

[Lazarsfeld 2004b] R. Lazarsfeld, Positivity in algebraic geometry, II: Positivity for vector bundles, and multiplier ideals, Ergebnisse der Math. und ihrer Grenzgebiete (3) 49, Springer, Berlin, 2004. MR 2005k:14001b Zbl 1093.14500

[Lazarsfeld and Mustaţă 2009] R. Lazarsfeld and M. Mustață, "Convex bodies associated to linear series”, Ann. Sci. Éc. Norm. Supér. (4) 42:5 (2009), 783-835. MR 2011e:14012 Zbl 1182.14004

[Okounkov 1996] A. Okounkov, "Brunn-Minkowski inequality for multiplicities", Invent. Math. 125:3 (1996), 405-411. MR 99a:58074 Zbl 0893.52004

[Okounkov 2003] A. Okounkov, "Why would multiplicities be log-concave?", pp. 329-347 in The orbit method in geometry and physics (Marseille, 2000), edited by C. Duval et al., Progr. Math. 213, Birkhäuser, Boston, 2003. MR 2004j:20022 Zbl 1063.22024

[Takayama 2006] S. Takayama, "Pluricanonical systems on algebraic varieties of general type", Invent. Math. 165:3 (2006), 551-587. MR 2007m:14014 Zbl 1108.14031

[Tsuji 2000] H. Tsuji, "Effective birationality of pluricanonical systems", preprint, 2000. arXiv math/0006166

Received May 10, 2010.

SHIN-YAO JOW

DEPARTMENT OF MATHEMATICS

UNIVERSITY OF PENNSYLVANIA

209 SOUTH 33RD STREET

PHILADELPHIA, PA 19104-6395

UNITED STATES

jows@math.upenn.edu 


\title{
PACIFIC JOURNAL OF MATHEMATICS
}

\author{
http://www.pjmath.org \\ Founded in 1951 by
}

E. F. Beckenbach (1906-1982) and F. Wolf (1904-1989)

\section{EDITORS}

V. S. Varadarajan (Managing Editor)

Department of Mathematics

University of California

Los Angeles, CA 90095-1555

pacific@math.ucla.edu

Vyjayanthi Chari

Department of Mathematics

University of California

Riverside, CA 92521-0135

chari@math.ucr.edu

\section{Robert Finn}

Department of Mathematics Stanford University

Stanford, CA 94305-2125

finn@math.stanford.edu

Kefeng Liu

Department of Mathematics

University of California

Los Angeles, CA 90095-1555

liu@math.ucla.edu
Darren Long

Department of Mathematics

University of California

Santa Barbara, CA 93106-3080

long@math.ucsb.edu

Jiang-Hua Lu

Department of Mathematics

The University of Hong Kong

Pokfulam Rd., Hong Kong jhlu@maths.hku.hk

Alexander Merkurjev

Department of Mathematics University of California

Los Angeles, CA 90095-1555 merkurev@math.ucla.edu
Sorin Popa

Department of Mathematics

University of California

Los Angeles, CA 90095-1555

popa@math.ucla.edu

Jie Qing

Department of Mathematics

University of California

Santa Cruz, CA 95064

qing@ cats.ucsc.edu

Jonathan Rogawski

Department of Mathematics

University of California

Los Angeles, CA 90095-1555

jonr@math.ucla.edu

\section{PRODUCTION}

pacific@math.berkeley.edu

Silvio Levy, Scientific Editor Matthew Cargo, Senior Production Editor

ACADEMIA SINICA, TAIPEI

CALIFORNIA INST. OF TECHNOLOGY

INST. DE MATEMÁTICA PURA E APLICADA

KEIO UNIVERSITY

MATH. SCIENCES RESEARCH INSTITUTE

NEW MEXICO STATE UNIV.

OREGON STATE UNIV.

\section{SUPPORTING INSTITUTIONS}

STANFORD UNIVERSITY
UNIV. OF BRITISH COLUMBIA
UNIV. OF CALIFORNIA, BERKELEY
UNIV. OF CALIFORNIA, DAVIS
UNIV. OF CALIFORNIA, LOS ANGELES
UNIV. OF CALIFORNIA, RIVERSIDE
UNIV. OF CALIFORNIA, SAN DIEGO
UNIV. OF CALIF., SANTA BARBARA

UNIV. OF CALIF., SANTA CRUZ

UNIV. OF MONTANA

UNIV. OF OREGON

UNIV. OF SOUTHERN CALIFORNIA

UNIV. OF UTAH

UNIV. OF WASHINGTON

WASHINGTON STATE UNIVERSITY

These supporting institutions contribute to the cost of publication of this Journal, but they are not owners or publishers and have no responsibility for its contents or policies.

See inside back cover or www.pjmath.org for submission instructions.

The subscription price for 2011 is US \$420/year for the electronic version, and \$485/year for print and electronic.

Subscriptions, requests for back issues from the last three years and changes of subscribers address should be sent to Pacific Journal of Mathematics, P.O. Box 4163, Berkeley, CA 94704-0163, U.S.A. Prior back issues are obtainable from Periodicals Service Company, 11 Main Street, Germantown, NY 12526-5635. The Pacific Journal of Mathematics is indexed by Mathematical Reviews, Zentralblatt MATH, PASCAL CNRS Index, Referativnyi Zhurnal, Current Mathematical Publications and the Science Citation Index.

The Pacific Journal of Mathematics (ISSN 0030-8730) at the University of California, c/o Department of Mathematics, 969 Evans Hall, Berkeley, CA 94720-3840, is published monthly except July and August. Periodical rate postage paid at Berkeley, CA 94704, and additional mailing offices. POSTMASTER: send address changes to Pacific Journal of Mathematics, P.O. Box 4163, Berkeley, CA 94704-0163.

PJM peer review and production are managed by EditFLOW ${ }^{\mathrm{TM}}$ from Mathematical Sciences Publishers.

PUBLISHED BY PACIFIC JOURNAL OF MATHEMATICS

at the University of California, Berkeley 94720-3840

A NON-PROFIT CORPORATION

Typeset in IATEX

Copyright $(2011$ by Pacific Journal of Mathematics 


\section{PACIFIC JOURNAL OF MATHEMATICS}

Volume $251 \quad$ No. $2 \quad$ June 2011

Two Kazdan-Warner-type identities for the renormalized volume coefficients and the Gauss-Bonnet curvatures of a Riemannian metric

BIN GUO, ZHENG-CHAO HAN and HAIZHONG LI

Gonality of a general ACM curve in $\mathbb{P}^{3}$

ROBIN HARTSHORNE and ENRICO SCHLESINGER

Universal inequalities for the eigenvalues of the biharmonic operator on submanifolds

SAÏD ILIAS and OLA MAKHOUL

Multigraded Fujita approximation

SHIN-YaO Jow

Some Dirichlet problems arising from conformal geometry

QI-RUi Li and WEIMIN SHENG

Polycyclic quasiconformal mapping class subgroups

KATSUHIKO MATSUZAKI

On zero-divisor graphs of Boolean rings

ALI MOHAMMADIAN

Rational certificates of positivity on compact semialgebraic sets

VICTORIA POWERS

Quiver grassmannians, quiver varieties and the preprojective algebra

Alistair Savage and Peter Tingley

Nonautonomous second order Hamiltonian systems

\section{MARTIN SCHECHTER}

Generic fundamental polygons for Fuchsian groups

AKIRA USHIJIMA

Stability of the Kähler-Ricci flow in the space of Kähler metrics

KAI ZHENG

The second variation of the Ricci expander entropy 